\title{
Factitious Lithium Toxicity Secondary to Lithium Heparin-Containing Blood Tubes
}

\author{
Brandon K. Wills, DO, MS ab, Mark B. Mycyk, MD ac, Suzan Mazor, MD ad, Michele Zell-Kanter, \\ PharmDa, Larry Brace, PhDe, Timothy Erickson, MDaf
}

\author{
a Toxikon Consortium: Cook County Hospital, University of Illinois at Chicago Medical Center, \\ Rush University Medical Center, and Illinois Poison Center, Chicago, IL \\ b Department of Emergency Medicine, Madigan Army Medical Center, Tacoma, WA \\ c Department of Emergency Medicine, Northwestern University School of Medicine, Chicago, IL \\ d Division of Emergency Medicine, Seattle Children's Hospital, Seattle, WA \\ e Department of Pathology, University of Illinois Medical Center, Chicago, IL \\ f Department of Emergency Medicine, University of Illinois Medical Center, Chicago, IL
}

\begin{abstract}
Introduction: Lithium concentrations analyzed from blood collected in inappropriate tubes may lead to misdiagnosis and lead to unnecessary hospitalization and intervention. We sought to assess the magnitude of falsely elevated lithium levels in green top lithium heparin-containing blood tubes.

Methods: Serum lithium concentrations from two types of commonly used standard green top tubes were evaluated against a control tube. Blood samples obtained from 5 healthy volunteers who have never ingested pharmaceuticals containing lithium were aliquoted into a control serum separator tube (SST), a light green heparin-containing tube, and a dark green heparin tube at the following volumes: full draw, 2cc, and 1cc. Serum lithium levels in lithium heparin blood tubes were compared to standard blood tubes.

Results: All levels are reported as $\mathrm{mmol} / \mathrm{L}$. Mean serum lithium level for the control was 0.16 [range: 0.1-0.2]. Levels for the light-green tubes at full-draw, 2cc, and 1cc were 1.05 [1.0-1.1], 1.99 [1.68-2.3], and 3.31 [2.8-4.2] respectively. Levels for the darkgreen tubes at full-draw, 2cc, and 1cc were 1.07 [1.0-1.14], 2.35 [2.1-2.55], and 4.04 [3.8-4.4] respectively.

Conclusions: Falsely elevated lithium levels may occur when using green-top lithium containing blood tubes and may contribute as much as $4 \mathrm{mmol} / \mathrm{L}$ to the level in tubes not completely filled.
\end{abstract}

\section{INTRODUCTION}

Over 5,000 lithium poisonings were reported to US poison centers in 2003 [1]. Patients being evaluated for suspected drug overdose or toxicity routinely have serum drug levels drawn for medications that may require specific interventions (e.g. antidote therapy, extracorporeal removal). Although it seems intuitive that lithium heparin- containing tubes (LHT) would be inappropriate when measuring serum lithium levels, such tubes are frequently used in cases of suspected toxicity to measure serum concentrations of several different medications. Our toxicology service was recently consulted to aid in an emergency department case of suspected

Keywords: lithium, overdose, poisoning, intoxication, laboratory analysis

Editor's Note: The opinion and assertions contained herein are the views of the author and are not to be construed as official or as reflecting the views of the United States Department of Defense.

Corresponding author: Brandon Wills, DO, MS, Madigan Army Medical Center Department of Emergency Medicine MCHJ-EM, Tacoma, WA 98431. Email: bkwills@gmail.com 
lithium intoxication in a 12-year-old patient after serum analysis demonstrated a toxic $(9.2 \mathrm{mmol} / \mathrm{L}$, normal $0.5-1.0)$ lithium level. In this case, the patient's lack of clinical signs and symptoms consistent with lithium toxicity did not correlate with the lithium level measured by our laboratory. Only after extensive clinical and laboratory evaluation was it determined that the lithium levels were falsely elevated due to LHT blood tubes. The delay to definitive diagnosis and potential for inappropriate invasive hemodialysis in this patient led us to perform a study to determine the variation in measured serum lithium levels that are a result of by tube type and volume of blood used.

\section{MATERIALS AND METHODS}

This descriptive study was performed to determine the contribution of lithium found in green top blood tubes to the reported serum lithium level. Institutional IRB approval was obtained. Two types of green top tubes-(1) Vacutainer ${ }^{\circledR} 13 \times 75 \mathrm{~mm}(4 \mathrm{~mL}$ draw, light green top) with serum separator gel and 57 USP units of lithium, and (2) Vacutainer® $13 \times 75 \mathrm{~mm}(5 \mathrm{~mL}$ draw, dark green top) with 72 USP units of lithium-were compared against a Vacutainer® serum separator tube (SST) that was used as a control. The control SSTs contained no lithium. Blood samples were obtained from 5 healthy volunteers who have never ingested lithium and who did not use pharmaceuticals, dietary supplements, vitamins, or illicit drugs. Blood samples were aliquoted in the following fashion: full-draw green top, 2cc, 1cc, and full-draw SST. Samples were analyzed using a Beckman Coulter SynchronCX7 system, colorimetric assay. Data were collected and entered into an Excel (Microsoft Corp., Redmond, WA) database and were analyzed using descriptive statistics.

\section{RESULTS}

All levels are reported as mmol/L. Mean serum lithium level for the control SST was 0.16 [range: 0.1-0.2]. Levels for the light-green tubes (gel) at full-draw, 2cc, and 1cc were 1.05 [1.0-1.1], 1.99 [1.68-2.3], and 3.31 [2.8-4.2] respectively. Levels for the dark-green tubes (no gel) at full-draw, 2cc, and 1cc were 1.07 [1.0-1.14], 2.35 [2.1-2.55], and 4.04 [3.8-4.4] respectively. Figure 1 displays the mean serum lithium levels by volume of blood used in each tube.

\section{DISCUSSION}

Our study demonstrates that the type of blood tube they are drawn in significantly affects lithium levels. While lithium levels in control tubes were non-detectable, there was an inversely proportional increase in the mean lithium level with the volume of blood in LHTs. Although it makes sense that lithium levels should not be measured from lithium containing green-top-tubes, such green-top-tubes are commonly used during the routine evaluation of overdose patients to measure the serum concentrations of a number of different pharmaceuticals. Since nurses and technicians frequently do blood draws, physicians are often unaware of the types of tubes used when interpreting results reported from a laboratory. Safe and appropriate management decisions rely on accurate results, especially when interventions such as hemodialysis may contribute to morbidity in patients who do not need such interventions.

Falsely elevated lithium levels from LHT blood tubes have been reported in two previous case reports [2,3]. Nordt and Cantrell reported a case of a 33-year-old woman with a multiple drug overdose. A falsely elevated lithium level was identified because of serum sampling in a lithium heparin tube [2]. In the case of multiple drug ingestion, detection of several drug levels was necessary and the single lithium heparin tube was convenient for all the levels except the lithium. Lee and Klachko described how an inaccurately high lithium level drawn in a lithium containing tube almost led to hemodialysis of a child 15-months of age [3]. Their case prompted them to perform a pilot study that demonstrated a measurable lithium level in serum collected in LHTs in non-lithium takers. Our study demonstrates that the vol-

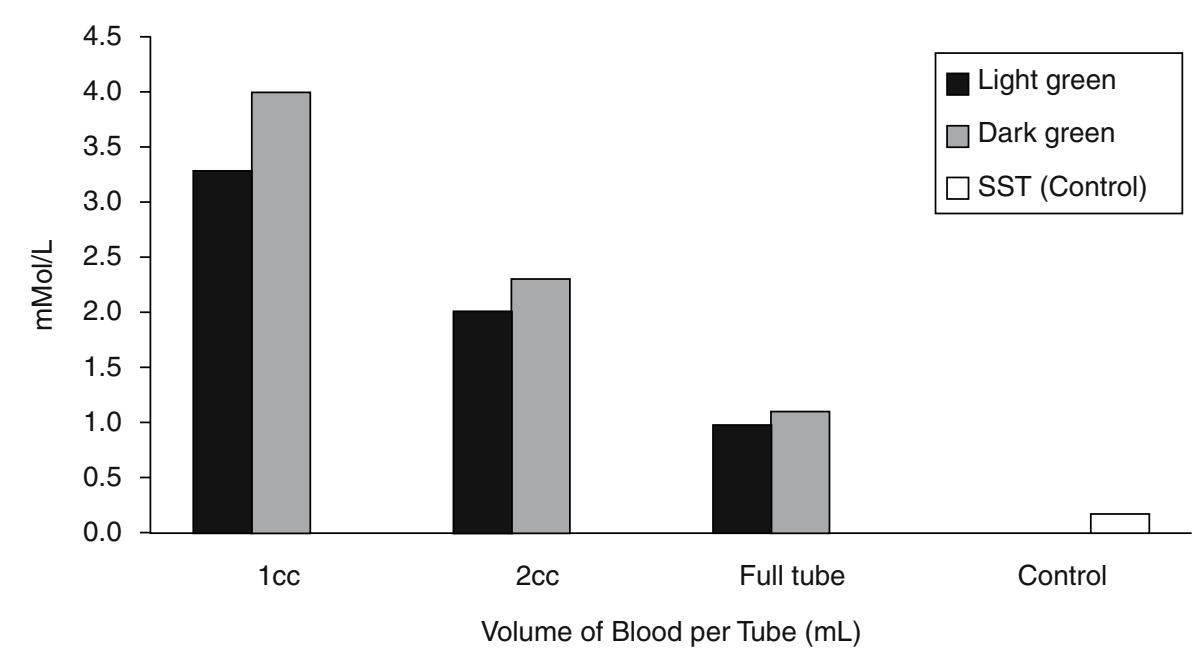

Figure 1. Mean serum lithium levels by volume of blood. 
ume of serum in LHTs is another clinically important contributing factor to the magnitude of falsely elevated lithium levels in subjects without any lithium exposure. Tubes that are incompletely filled can add as much as $4 \mathrm{mmol} / \mathrm{L}$ to the reported level.

Due to lithium containing blood tubes, lithium levels in those two reports ranged from $1.5-3.4 \mathrm{mmol} / \mathrm{L}$. The volunteers in our study who did not take lithium demonstrated that the potential exists for a $1 \mathrm{mmol} / \mathrm{L}$ elevation from a full blood draw in a lithium containing tube and that increases of up to $4 \mathrm{mmol} / \mathrm{L}$ may result from the inadequate filling of tubes.

\section{LIMITATIONS}

Our study does not address to what degree lithium levels drawn in LHT impacts patients taking lithium either therapeutically or in overdose.

\section{CONCLUSION}

Falsely elevated lithium levels that suggest significant toxicity may occur when using lithium containing blood tubes. Although these tubes are appropriate for other assays, healthcare staff should be educated concerning the potential for diagnostic errors in cases where lithium toxicity is being evaluated.

The authors have no potential financial conflicts of interest to report.

\section{REFERENCES}

1. Watson W, Litovitz T, Klein-Schwartz W, et al. 2003 annual report of the American Association of Poison Control Centers Toxic Exposure Surveillance System. Am J Emerg Med 2004; 22(5):335-404.

2. Nordt S, Cantrell L. Elevated Lithium Level: A Case and Brief Overview of Lithium Poisoning. Psychosom Med 1999; 61(4):564-565.

3. Lee D, Klachko M. Falsely Elevated Lithium Levels in Plasma Samples Obtained in Lithium Containing Tubes. J Toxicol Clin Toxicol 1996; 34(4):467-469. 